\title{
Über eine Verallgemeinerung der Legendre-Hadamardschen Bedingung auf restringierte Variationsprobleme ${ }^{\text {I) }}$
}

\author{
R. KLÖTZLER
}

\begin{abstract}
Variational problems are studied with continuous integrands, subject to vector-valued functions $x$ of vector varlables, $t$ with restricted derivatives $x_{t}$, under some goodness conditions of their feasible set $\dot{X}$. For an optimal solution $x_{0}$ it will be shown the necess ity of the (strong) quasi-convexity (in the sense of C.B. Morrey) of the integrand on $\dot{X}$ ii) regard of $\lambda_{0}$.
\end{abstract}

Key words: Legendre-Hadamardsche Bedingung, q-Konvexität

AMS subject classification: $49 \dot{B} 36$

\section{Einleitung}

Für mehrdimensionale Variationsprobleme auf einem Integrationsgebiet $\Omega \subset \mathbb{E}^{m}$ in der Form

$$
\begin{aligned}
& J(x)=\int_{\Omega} f\left(t, x(t), x_{t}(t)\right) d t \rightarrow \text { Min auf } C^{1, n}(\bar{\Omega}) \\
& \text { unter Randbedingungen } x=\varphi_{0} \text { auf } \partial \Omega
\end{aligned}
$$

stellt die Legendre-Hadamardsche Bedingung ein klassisches notwendiges Optimalitätskriterium dar. Ist $x_{0}$ eine optimale Lösung von (0) und $f \in C^{2}$, so muß nach J. HADAMARD [3] in allen Punkten $t \in \Omega$ die Ungleichung

$$
f_{x_{t \alpha}^{i} x_{i \beta}}\left(t, x_{0}(t), x_{\alpha_{t}}(t)+\zeta \lambda^{\top}\right) \zeta_{j} \zeta_{j} \lambda^{\alpha} \lambda^{\beta} \geq 0
$$

für beliebige (Spalten-) Vektoren $\lambda \in \mathbb{E}^{m}, \zeta \in \mathbb{E}^{n}$ gelten. Hierbei wird in dieser (und auch in nachfolgender) Darstellung iber doppelt auftretende Indizes summiert $; \lambda^{\top}=\left(\lambda^{1}, \ldots, \lambda^{m}\right)$, $\zeta^{\top}=\left(\zeta_{1}, \ldots, \zeta_{n}\right)$ und $x_{t}$ wird als $(n \times m)$-Matrix der partiellen Ableitungen $x_{t \alpha}^{i}$ aufgefaßt. Da in dieser Bedingung Ableitungen zweiter Ordnung von $f$ benutzt werden, nennen wir sie Legendre-Hadamardsche Bedingung 2. Ordnung.

Ist hingegen $f \in C^{1}$, so muß nach L.M. Graves [2] in allen $t \in \Omega$ die Ungleichung

$$
\begin{aligned}
& f\left(t, x_{0}(t), x_{o_{t}}(t)+\zeta \lambda^{\top}\right) \\
& \quad \geq f\left(t, x_{0}(t), x_{o_{t}}(t)\right)+f_{x_{i}^{i} \alpha}\left(t, x_{o}(t), x_{o_{t}}(t)\right)\left(\lambda^{\alpha} \zeta_{i}\right) \quad \forall \lambda \in \mathrm{E}^{m}, \zeta \in \mathrm{E}^{n}
\end{aligned}
$$

erfullt sein: Diese Forderung heißt WeierstraB-Bedingung; wir nennen sie auch LegendreHadamardsche Bedingung 1. Ordnung.

1) Dieser Beitrag stellt eine Erweiterung der Untersuchung [5] dar. Sie entstand im Rahmen eines Studienaufenthaltes an der Rheinischen Friedrich-Wilhelm-Universität Bonn mit dankenswerter Unterstiutzung des Sonderforschungsbereiches 256. 
Ist schließlich $f \in C$, so werden wir zeigen, daß in allen Punkten $t \in \Omega$ die Beziehung

$$
\Phi\left[t, x_{0} ; \Lambda, \zeta, H\right] \equiv \sum_{k=0}^{m} f\left(t, x_{0}(t), x_{0, t}(t)+\zeta \lambda_{k}^{\top}\right) \mu_{k}-f\left(t, x_{0}(t), x_{0}(t), x_{0 t}(t)\right) \geq 0
$$

auf der Menge

$$
K=\left\{(\Lambda, \zeta, \mu) \mid \begin{array}{l}
\Lambda=\left(\lambda_{0}, \ldots, \lambda_{m}\right), \zeta \in \mathbb{E}^{n}, \mu=\left(\mu_{0}, \ldots, \mu_{m}\right) \\
\mu_{k} 20 \quad(k=0, \ldots, m), \sum_{k=0}^{m} \mu_{k}=1, \sum_{k=0}^{m} \mu_{k} \lambda_{k}=0
\end{array}\right\}
$$

gelten muB. Wir nennen (3) auch die Legendre-Hadamardsche Bedingung 0 . Ordnung.

Aus (1) folgt (2) und aus (2) folgt (3). Man zeigt auch leicht, daB unter der Voraussetzung $f \in C^{1}$ aus (3) die Bedingung (2) folgt. Ist $f$ sogar aus $C^{2}$, so resultiert (1) aus (2). Man vergleiche dazu z.B. C.B. MorREy [6: S.112]. Für $m=1$ wurde die Bedingung (3) bereits durch L. BITTNER [1] hergeleitet.

Das Hauptanliegen dieser Arbeit besteht einerseits darin, unter gewissen Einschränkungen die Notwendigkeit der Bedingungen (1), (2) und (3) auch dann noch nachzuweisen, wenn unser Variationsproblem zusätzliche Nebenbedingungen der Gestalt

$$
x_{t}(t) \in \dot{X}(t, x(t)) \text { für fast alle } t \in \Omega
$$

erhält. Zum anderen werden wir eine Verallgemeinerung der Bedingung (3) herleiten, die in enger Beziehung zur "starken Quasikonvexität" im Sinne von C. B. MORREY [6] steht. Wir setzen dabei die mengenwertige Abbildung $\dot{X}: \bar{\Omega} \times \mathbb{E}^{n} \rightarrow \mathbb{E}^{n m}$ als stetig voraus. Außerdem sei

$$
\dot{X}(t, \xi)=\overline{\text { int } \dot{X}(t, \xi)} \neq \Phi \text { für alle }(t, \xi) \in \bar{\Omega} \times \mathbb{E}^{n} .
$$

Mit diesem Ergebnis werden wichtige Voraussetzungen geschaffen, die im Sinne von $R$. KLÖTZLER [4] eine Erweiterung des Pontryaginschen Maximumprinzips auf Steuerungs- und Variationsprobleme mit mehrfachen Integralen ermöglichen.

\section{Vorbereitende Begriffo und Bezeichnungen}

In dieser Arbeit wird mit $B(w, \varepsilon)$ zu $w \in \mathbb{E}^{r}$ und $\varepsilon>0$ die offene Kugel des $\mathbb{E}^{r}$ mit dem Mittelpunkt $w$ und Radius $\varepsilon$ bezeichnet. Ferner soll dist $(A, w)$ der euklidische Abstand des Punktes $w$ von der Menge $A \subset \mathbb{E}^{r}$ sein. Ist $A$ eine Menge des $\mathbb{E}^{r}$, so ist

$$
A_{\varepsilon}:=\left\{w \in \mathbb{E}^{r} \mid \operatorname{dist}(A, w)<\varepsilon\right\} \text { und } A_{-\varepsilon}:=\{w \in A \mid \operatorname{dist}(\partial A, w)>\varepsilon\} \text {, }
$$

wobei $\partial A$ den Rand von $A$ bezeichnet.

Zu einem System $\Lambda=\left\{\lambda_{0}, \ldots, \lambda_{m}\right\}$ von $m+1$ Vektoren $\lambda_{0}, \ldots, \lambda_{m}$ des $\mathbb{E}^{m}$ bezeichne $S(\Lambda)$ das Simplex mit den Eckpunkten $\lambda_{0}, \ldots, \lambda_{m}$. Es heißt regulär, wenn es innere Punkte enthält, anderenfalls heißt es nichtregulär .

Eine Funktion $g$ vo.n $(n \times m)$-Matrizen $w=\left(w_{\alpha}^{i}\right)_{\alpha=1, \ldots, m}^{j=1, \ldots, n}$ heißt nach C.B. MorREY [6: S. 112] quasikonvex - wir sagen dafür kurz $q$-konvex - wenn für beliebige Matrizen $w$ dieser Art und $(\Lambda, \zeta, \mu)$ aus $K$ gemäB (3') die Ungleichung $\sum_{k=0}^{m} g\left(w+\zeta \lambda_{k}^{\top}\right) \mu_{k} \geq g(w)$ gilt. Eine Funktion $g$ von $(n \times m)$-Matrizen $w$ heißt nach C.B. MORREY [6:S.114] stark 
quasikonvex - wir sagen dafür kurz stark $q$-konvex - wenn für beliebige beschränkte Gebiete $U \subset \mathbb{E}^{m}$ und $n$-vektorwertige Lipschitz-stetige Funktionen $z$ auf $U$ mit der Eigenschaft supp $z \subset U$ die Ungleichung

$$
\int_{U} g\left(w+z_{t}(t)\right) d t z g(w) \operatorname{mes} U
$$

gilt.

Eine stetige mengenwertige Abbildung $\chi$ von Punkten $z \in \mathbb{E}^{l}$ in den $\mathbb{E}^{r}$ ist nach $S$. ROLEWICZ [7] durch die folgenden zwei Eigenschaften charakterisiert:

a) Für alle $\varepsilon>0$ existiert ein $\delta(\varepsilon)$ mit $\chi\left(z_{0}\right)_{\varepsilon} \supset \chi(z)$ für alle $z \in B\left(z_{0}, \delta(\varepsilon)\right)$.

b) Für alle $\varepsilon>0$ existiert ein $\delta(\varepsilon)$ mit $\chi\left(z_{0}\right) \supset \chi(z)_{\varepsilon}$ für alle $z \in B\left(z_{0}, \delta(\varepsilon)\right)$.

Setzen wir speziell in (5) für das Argument $\xi$ den Wert $x(t)$ für $x \in C^{\alpha, n}(\bar{\Omega})$ ein, so wird $\chi(\cdot)=\dot{X}(\cdot, x(\cdot))$ eine stetige mengenwertige Funktion auf $\bar{\Omega}$.

\section{Anwendung einer allgemeinen Klasse von Variationen}

Im Gegensatz zu L. M. Graves [2] verwenden wir hier für die Herleitung von (3) eine allgemeinere Technik, die es auch gestattet, Nebenbedingungen der Gestalt (4) einzubeziehen. Wir setzen dabei lediglich $f \in C$ voraus. Wir studieren nun das Variationsproblem $(0)$ unter der Nebenbedingung (4) auf dem allgemeineren Funktionenraum $W_{q}^{1, n}(\Omega)$ mit $q>m$, d.h.

$$
I(x)=\int_{\Omega} f\left(t, x(t), x_{t}(t)\right) d t \rightarrow \operatorname{Min} \text { auf } W_{q}^{1, n}(\Omega)
$$

unter der Nebenbedingung $x_{\mathrm{f}}(t) \in \dot{X}(t, x(t))$ fast überall auf $\Omega$ und Randbedingung $x=\varphi_{0}$ auf $\partial \Omega$.

Hypothese: Das Problem (6) besitzt eine Lösung $x_{0} \in W_{q}^{1, n}(\Omega)$, für die $x_{o_{t}}$ fast überall stetig auf $\Omega$ ist.

Nach den Sobolevschen Einbettungssätzen ist zunächst $x_{0}$ stetig auf $\bar{\Omega}$. Dann bilden wir unter obiger Hypothese zu einem beliebigen Stetigkeitspunkt $t_{0} \in \Omega$ von $x_{0 t}$ mit der Eigenschaft

$$
x_{o_{t}}\left(t_{0}\right) \in \operatorname{int}\left[\dot{X}\left(t_{o}, x_{0}\left(t_{0}\right)\right)\right]
$$

eine spezielle Folge von Störfunktionen $\left\{\xi^{k}\right\}_{k \in N}$. Dazu greifen wir uns eine beliebige Umgebung $U \subset \Omega$ von $t_{0}$ heraus sowie eine beliebige Lipschitz-stetige $n$-vektorwertige Funktion $\varphi$ auf $\Omega$ mit den Eigenschaften

$$
\operatorname{supp} \varphi \subset U, x_{0_{t}}\left(t_{0}\right)+\varphi_{t}(t) \in \operatorname{int}\left[\dot{X}\left(t_{0}, x_{0}\left(t_{0}\right)\right)\right] .
$$

Wegen (7) ist die Menge dieser $\varphi$ nicht leer. Außerdem erklären wir

$$
U_{k}=\left\{t \in \Omega \mid t_{0}+k\left(t-t_{0}\right) \in U\right\}
$$

und

$$
\xi^{k}(t)= \begin{cases}0 & \text { für } t \in U_{k} \\ k^{-1} \varphi\left(t_{0}+k\left(t-t_{0}\right)\right) & \text { für } t \in U_{k}\end{cases}
$$


Diese Funktionenfolge $\left\{\xi^{k}\right\}$ hat die Eigenschaft, ähnliche Graphen mit dem Ähnlichkeitszentrum $\left(t_{0}, 0\right)$ zu besitzen. Wegen der Bildstetigkeit von $\dot{X}$ existiert zu $\varphi$ ein $k_{0}$, so daß infolge (8) auch

$$
x_{\mathrm{o}_{t}}(t)+\xi_{t}^{k}(t) \in \operatorname{int}\left[\dot{X}\left(t, x_{\mathrm{o}}(t)\right)\right] \text { für } k \geq k_{\mathrm{o}} \text { und } t \in U_{k}
$$

gilt. Infolgedessen ist

$$
I\left(x_{0}+\xi^{k}\right)-I_{0}\left(x_{0}\right) \geq 0 \text { für alle } k \geq k_{0} .
$$

Unter Ergänzung von geeigneten Summanden können wir diese Differenz auch folgendermaßen schreiben:

$$
\begin{aligned}
& I\left(x_{0}+\xi^{k}\right)-I_{0}\left(x_{0}\right)=\int_{U_{k}}\left[f\left(t, x_{0}(t)+\xi^{k}(t), x_{o_{t}}(t)+\xi_{t}^{k}(t)\right)-f\left(t, x_{0}\left(t_{0}\right), x_{o_{t}}\left(t_{0}\right)+\xi_{t}^{k}(t)\right)\right] d t \\
& -\int_{U_{k}}\left[f\left(t, x_{0}(t), x_{\mathrm{o}_{\tau}}(t)\right)-f\left(t_{\mathrm{o}}, x_{\mathrm{o}}\left(t_{\mathrm{o}}\right), x_{\mathrm{o}_{t}}\left(t_{\mathrm{o}}\right)\right)\right] d t \\
& +\int_{U_{k}}\left[f\left(t_{0}, x_{0}\left(t_{0}\right), x_{\mathrm{o}_{t}}\left(t_{0}\right)+\xi_{t}^{k}(t)\right)-f\left(t_{0}, x_{0}\left(t_{0}\right), x_{\mathrm{o}_{t}}\left(t_{0}\right)\right)\right] d t .
\end{aligned}
$$

Die ersten beiden Integrale der rechten Seite von Gleichung (13) sind wegen der Stetigkeit von $f$ von der Größenordnung (mes $U_{k}$ ) $o(k)$. Der Ausdruck

$$
\frac{1}{\operatorname{mes} U_{k}} \int_{U_{k}}\left[f\left(t_{0}, x_{0}\left(t_{0}\right), x_{0 \ell}\left(t_{0}\right)+\xi_{t}^{k}(t)\right)-f\left(x_{0}, x_{0}\left(t_{0}\right), x_{0}\left(t_{0}\right)\right)\right] d t
$$

ist unabhängig von $k$. Denn unter Beachtung von (9) und (10) können wir den Ausdruck (14) mittels der Substitution $\tau=k\left(t-t_{0}\right)+t_{0}$ umformen in

$$
\frac{k^{-m}}{\operatorname{mes} U_{k}} \int_{U_{k}}\left[f\left(t_{0}, x_{0}\left(t_{0}\right), x_{0}\left(t_{0}\right)+\varphi_{\tau}(\tau)\right)-f\left(t_{0}, x_{0}\left(t_{0}\right), x_{0}\left(t_{0}\right)\right)\right] d \tau
$$

und der Faktor vor diesem Integral ist gleich $1 /$ mes $U$. Somit erhalten wir aus (13) nach Division mit $1 /$ mes $U_{k}$ und Grenzübergang $k \rightarrow \infty$ wegen (12) die notwendige Bedingung

$$
\int_{U} f\left(t_{0}, x_{0}\left(t_{0}\right), x_{0_{t}}\left(t_{0}\right)+\varphi_{\tau}(\tau)\right) d \tau 2(\operatorname{mes} U) f\left(t_{0}, x_{0}\left(t_{0}\right), x_{0 \tau}\left(t_{0}\right)\right) .
$$

Zusammengefaßt lautet unser Resultat:

Satz 1: Ist $x_{0}$ eine Lösung des restringierten Variationsproblems (6), so muB in allen Stetigkeitspunkten $t_{0} \epsilon \Omega$ von $x_{o_{t}}$ mit der Eigenschaft (7) die Bedingung (15) gelten $z u$ beliebigen Lipschitz-stetigen $n$-vektorwertigen Funktionen $\varphi$, die den Einschränkungen (8) genügen. 


\section{Spezielle Folgerungen}

Wir setzen in Anlehnung an [5] zu einem gegebenen regulären $m$-Simplex $S(\Lambda)$ mit den Eckpunkten $\lambda_{j} \in \mathbb{E}^{m}(j=0, \ldots, m)$ und $0 \in$ int $S(\Lambda)$ in (15) die Funktion $\varphi$ in der speziellen Gestalt

$$
\varphi(t)=-\zeta \operatorname{Max}\left\{0, \delta-\operatorname{Max}\left(\lambda_{j}^{\top}\left(t-t_{0}\right)\right)\right\}
$$

an. Dabei ist $\zeta$ ein beliebiger Vektor des $\mathbb{E}^{n}, \Lambda=\left(\lambda_{0}, \ldots, \lambda_{m}\right)$ und $\delta>0$ eine so kleine Zahl, daß supp $\varphi \subset \Omega$ gilt. Für jeden Index $i=1, \ldots, n$ bilden dann die Graphen von $\varphi^{i}$ pyramidenförmige Ausbeulungen der Ebene mit der Pyramidenbasis

$$
\left.U=\Delta:=\left\{t \in \mathrm{E}^{m} \mid \lambda_{j}^{r}\left(t-t_{0}\right) \leq \delta, j=0, \ldots, m\right)\right\}
$$

und der Pyramidenhöhe $-\zeta^{i} \delta$. Die Forderung (8) äußert sich jetzt in den Bedingungen

$$
x_{o_{t}}\left(t_{0}\right)+\zeta \lambda_{j}^{\top} \in \operatorname{int} \dot{X}\left(t_{0}, x_{0}\left(t_{0}\right)\right) \quad(j=0, \ldots, m) .
$$

Die Projektion der $j$-ten Pyramidenseite auf die $t$-Hyperebene bezeichnen wir mit $\Delta_{j}$, so daß $\Delta=\bigcup_{j=0}^{m} \Delta_{j}$ ist. Außerdem ist $\varphi_{t}$ auf jedem $\Delta_{j}$ konstant. Die nach außen orientierte Plangröße der $j$-ten Pyramidenseite beträgt $\mathfrak{n}_{j}$ mit

$$
\mathfrak{n}_{t}^{\dagger}=\operatorname{sgn} \zeta^{i}\left(+\zeta^{i} \lambda_{j}^{1}, \ldots,+\zeta^{i} \lambda_{j}^{m},-1\right) \operatorname{mes} \Delta_{j}(j=0, \ldots, m) .
$$

Die nach außen orientierte Plangröße der Pyramidenbasis ist $\operatorname{der}(m+1)$-dimensionale Vektor $\boldsymbol{n}$ mit $\mathfrak{n}^{\gamma}=(0, \ldots, 0,+$ mes $\Delta)$ sgn $\zeta^{j}$. Da die Summe der Plangröße eines Polyeders bekanntlich immer gleich dem Nullvektor ist, gilt neben mes $\Delta=\sum_{j=0}^{m}$ mes $\Delta_{j}$ zugleich

$$
\sum_{j=0}^{m} \lambda_{j} \operatorname{mes} \Delta_{j}=0 .
$$

Mit $\mu_{j}:=$ mes $\Delta_{j} /$ mes $\Delta$ geht dann die Bedingung (15) in

$$
\sum_{j=0}^{m} \mu_{j} f\left(t_{0}, x_{0}\left(t_{0}\right), x_{0 t}\left(t_{0}\right)+\zeta \lambda_{j}^{\top}\right) \geq f\left(t_{0}, x_{0}\left(t_{0}\right), x_{O_{t}}\left(t_{0}\right)\right)
$$

über. Dabei ist

$$
\sum_{j=0}^{m} \mu_{j}=1, \mu_{j}=0 \text { und } \sum_{j=0}^{m} \mu_{j} \lambda_{j}=0 .
$$

Die Bedingung (19) haben wir vorerst unter den Einschränkungen $0 \in \operatorname{int} S(\Lambda)$ und (17) erhalten. Da aber ein nichtreguläres $m$-Simplex beliebig genau durch reguläre $m$-Simplexe approximiert werden kann, gilt letztlich wegen (5) die Bedingung (19) auch für beliebige $\Lambda$ mit $0 \in S(\Lambda)$ und

$$
x_{0}\left(t_{0}\right)+\zeta \lambda_{j}^{\top} \in \dot{x}\left(t_{0}, x_{0}\left(t_{0}\right)\right) \text {. }
$$

Unsere Ergebnisse fassen wir in folgendem Satz zusammen.

Satz 2: Ist $x_{0}$ eine Lösung des restringierten Variationsproblems (6), so muB in allen Stetigkeitspunkten $t_{0} \in \Omega$ von $x_{0}$ mit der Eigenschaft (7) die Bedingung (19) gelten $z u$ beliebigen $\zeta \in \mathbb{E}^{n}, \lambda_{j} \in \mathbb{E}^{m}$ und $\mu_{j}(j=0, \ldots, m)$, die den Einschränkungen (20) und (21). genügen. 
Offensichtlich deckt sich im Falle des Fehlens von Nebenbedingungen in (6), d.h. $\dot{X}=$ $\mathbb{E}^{n ! m}$, die Aussage des Satzes 2 mit der Legendre-Hadamardschen Bedingung 0-ter Ordnung gemäß (3).

Ist die Bedingung (3) für beliebige dritte Argumente $p_{0}=x_{0 t}$ erfullt, so nennt C.B.MoRREY [6: S. 112] die Funktion $f\left(t, x_{0}(t), \cdot\right)$ quasikonvex. Da dieser Begriff in der Optimierungstheorie aber noch in gänzlich davon abweichendem Sinne gebraucht wird, wollen wir besser $q$-konvex sagen (vgl. auch Abschnitt 2). Ist die Bedingung (15) für beliebige dritte Argumente $p_{0}=x_{0}$ erfüllt, so nennt C. B. MORREY [6: S. 114] die Funktion $f\left(t_{0}, x_{0}\left(t_{0}\right), \cdot\right)$ stark quasikonvex. Wir wollen hierfür wieder stark q-konvex sagen.

Im Hinblick auf unsere restringierten Variationsprobleme (6) wollen wir hier und in Nachfolgearbeiten die durch Satz 2 gefolgerte Optimalitätsbedingung (19) als

$$
q \text {-Konvexität von } f\left(t_{0}, x_{0}\left(t_{0}\right), \cdot\right) \text { auf } \dot{X}\left(t_{0}, x_{0}\left(t_{0}\right)\right) \text { bezüglich } x_{0 t}\left(t_{0}\right)
$$

bezeichnen. In entsprechender Weise drücken wir die durch Satz 1 geforderte Bedingung (15) als

starke $q$-Konvexität von $f\left(t_{0}, x_{0}\left(t_{0}\right), \cdot\right)$ auf $\dot{X}\left(t_{0}, x_{0}\left(t_{0}\right)\right)$ bezüglich $x_{0_{p}}\left(t_{0}\right)$ aus.

\section{LITERATUR}

[1] BITTNER, L.: A remark concerning the Weierstrass condition without derivatives. Preprint. Greifswald: Ernst-Moritz-Arndt-Universität, Preprint-Reihe Math. 13 (1985). $1-4$.

[2] GRAVES, L. M.: The Weierstraß condition for multiple integral variation problem. Duke Math J. 5 (1939), 656 - 660.

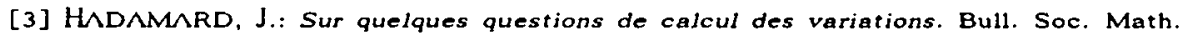
France 33 (1905), 73 - 80.

[4] KLOTZLER, R.: Exiensions of Pontryagin's Maximum Principle. In: Proceedings of the $9^{\text {th }}$ IFIP-Conference Leipzig 1989 (to appear).

[5] KLÖTZLER, R.: Uber eine Verallgemeinerung der Legendre-Hadamardschen Bedingung auf restringierte Variationsprobleme. Preprint. Bonn: Preprint-Reihe dos Sonderforschungsbereichs 256 der Universitat Nr. 87 (1989), $1-12$.

[6] MORREY, C. B.: Multiple Integrals in the Calculus of Variations. Berlin * Heidelberg - New York: Springer-Verlag 1966.

[7] ROLEWICZ, S.: Funktionalanalysis und Steuerungstheorie. Berlin-Heidelberg - New York: Springer-Verlag 1976.

Received 20.02.1990

Prof. Dr. Rolf Klözler

Sektion Mathematik der Universität Leipzig

Augustusplatz 10

D (Ost) - 7010 Leipzig 